\title{
Structural Effects of Disease-Related Mutations in Actin-Binding Period 3 of Tropomyosin
}

\author{
Balaganesh Kuruba ${ }^{1}$, Marta Kaczmarek ${ }^{2}$, Małgorzata Kęsik-Brodacka ${ }^{3}$, Magdalena Fojutowska ${ }^{2}$, \\ Małgorzata Śliwinska ${ }^{2}$, Alla S. Kostyukova ${ }^{1}$ and Joanna Moraczewska ${ }^{2, *}$
}

1 Gene and Linda Voiland School of Chemical Engineering and Bioengineering, Washington State University, Pullman, WA 99163, USA; balaganesh.kuruba@wsu.edu (B.K.); alla.kostyukova@wsu.edu (A.S.K.)

2 Department of Biochemistry and Cell Biology, Faculty of Biological Sciences, Kazimierz Wielki University, 85-671 Bydgoszcz, Poland; marta92@ukw.edu.pl (M.K.); magdalena.fojutowska@ukw.edu.pl (M.F.); gosia.sl@ukw.edu.pl (M.Ś.)

3 National Medicines Institute, 00-725 Warsaw, Poland; m.kesik@nil.gov.pl

* Correspondence: joanna.moraczewska@ukw.edu.pl; Tel.: +48-52-325-9219

\section{check for} updates

Citation: Kuruba, B.; Kaczmarek, M.; Kęsik-Brodacka, M.; Fojutowska, M.;

Śliwinska, M.; Kostyukova, A.S.;

Moraczewska, J. Structural Effects of

Disease-Related Mutations in

Actin-Binding Period 3 of

Tropomyosin. Molecules 2021, 26, 6980. https://doi.org/10.3390/

molecules 26226980

Academic Editors: Matthew Merski and Dirk Linke

Received: 12 October 2021

Accepted: 16 November 2021

Published: 19 November 2021

Publisher's Note: MDPI stays neutral with regard to jurisdictional claims in published maps and institutional affiliations.

Copyright: (c) 2021 by the authors. Licensee MDPI, Basel, Switzerland. This article is an open access article distributed under the terms and conditions of the Creative Commons Attribution (CC BY) license (https:// creativecommons.org/licenses/by/ $4.0 /)$.

\begin{abstract}
Tropomyosin (Tpm) is an actin-binding coiled-coil protein. In muscle, it regulates contractions in a troponin $/ \mathrm{Ca}^{2+}$-dependent manner and controls the thin filament lengths at the pointed end. Due to its size and periodic structure, it is difficult to observe small local structural changes in the coiled coil caused by disease-related mutations. In this study, we designed 97-residue peptides, Tpm1.1 $1_{64-154}$ and Tpm3.12 $65-155$, focusing on the actin-binding period 3 of two muscle isoforms. Using these peptides, we evaluated the effects of cardiomyopathy mutations: I92T and V95A in Tpm1.1, and congenital myopathy mutations R91P and R91C in Tpm3.12. We introduced a cysteine at the N-terminus of each fragment to promote the formation of the coiled-coil structure by disulfide bonds. Dimerization of the designed peptides was confirmed by gel electrophoresis in the presence and absence of dithiothreitol. Using circular dichroism, we showed that all mutations decreased coiled coil stability, with Tpm3.1265-155 R91P and Tpm1.164-154 I92T having the most drastic effects. Our experiments also indicated that adding the N-terminal cysteine increased coiled coil stability demonstrating that our design can serve as an effective tool in studying the coiled-coil fragments of various proteins.
\end{abstract}

Keywords: tropomyosin; peptide; stability; congenital myopathy; cardiomyopathy; point mutations; coiled coil; circular dichroism; molecular dynamics simulation

\section{Introduction}

Tropomyosin $(\mathrm{Tpm})$ is a protein belonging to a family of actin regulatory proteins which bind to actin filaments to control interactions of actin with its binding partners. Two $\alpha$-helical molecules of Tpm fold into a coiled coil and polymerize end-to-end to form uninterrupted chains along the entire length of actin filaments [1]. In response to fluctuations in sarcoplasmic $\mathrm{Ca}^{2+}$ concentration in striated muscles, Tpm together with troponin complex (Tn), regulates the actin-myosin interactions, and, thus, the contraction of muscle fibers [2]. In the contractile apparatus of different types of mammalian striated muscle, three isoforms of Tpm were found: Tpm1.1 expressed in cardiac and fast skeletal muscle, Tpm 2.2 in cardiac, slow and fast muscle, and Tpm3.12 that is expressed exclusively in slow muscle fibers. The isoforms are products of TPM1, TPM2, and TPM3 genes, respectively [3]. Although Tpm1.1 and Tpm3.12 are highly homologous proteins showing $91 \%$ identity and $\sim 96 \%$ sequence similarity [4], their regulatory functions are quantitatively differentiated $[5,6]$. However, it is not known, which sequence differences are responsible for the regulatory performance of the isoforms. As a coiled-coil protein, the Tpm sequence is characterized by the presence of regular repeats of seven amino acid residues (labeled from $a$ to $g$ ) known as heptad repeats. Hydrophobic amino acid residues, such as Val, Ile, Leu, 
are frequently present in the 1 st $(a)$ and 4 th $(d)$ positions of each repeat. Due to the $\alpha$-helical fold of each Tpm chain, the hydrophobic residues are exposed on one face of the $\alpha$-helix, which allows for locking them with the hydrophobic residues from the second chain in "knobs into holes" fashion. This forms a hydrophobic core necessary for dimerization and stabilization of two chains in the coiled-coil structure. Electrostatic, interhelical interactions between residues in $e$ and $g$ positions provide additional stabilization. In addition to the stabilizing hydrophobic residues, the core of Tpm coiled coil harbors clusters of small hydrophobic or polar residues such as alanine and serine, which cause bending and local flexibility thought to be necessary for Tpm to wrap around actin filaments [7,8].

The second periodic pattern along the Tpm sequence is related to its interactions with seven consecutive actin subunits. Each actin-binding period contains charged residues which are exposed on the outer surface of the coiled coil in $b, c$, and $f$ positions of the heptad repeat [8]. Electrostatic interactions with oppositely charged residues on actin subunits mostly determine the interface between Tpm and actin $[9,10]$. Although the actin-binding periods are regarded as quasi-equivalent, they bind to actin with different affinities [11,12]. In addition to interactions with actin, the actin-binding periods have specific tasks, such as end-to-end interactions, binding TnT and TnI (the subunits of Tn complex), and interactions with the thin filament capping proteins and with myosin heads $[13,14]$. Several key structural features of Tpm periods are evolutionarily conserved and determine Tpm interactions with binding partners, stability, and flexibility [9,15-19].

A very conservative region of Tpm is the internal actin-binding period 3, the only period that is identical in the three striated muscle isoforms. A few amino acid differences between muscle isoforms are present in the close vicinity of period 3, but it is not known if these differences have any influence on period 3 structure. The deletion of the entire period 3 mildly impaired the binding of Tpm to F-actin in the presence of $\mathrm{Tn}\left(+\mathrm{Ca}^{2+}\right)[20]$, but severely affected cooperative allosteric changes in the filament that are important for interactions of myosin heads with the filament and development of isometric force [11,21]. The deletion of the Period 3 mutant drastically reduced the activation of the velocity of reconstituted thin filament movements on myosin head-covered surfaces [22]. This implies that structural changes within period 3 can be responsible for hypocontractile phenotype and muscle weakness. Nevertheless, point mutations found in humans in this region of Tpm are responsible not only for hypocontractile phenotypes related to reduced activation but also to hypercontractile phenotypes characterized by the increased activation of actin-activated myosin ATPase $[23,24]$. It is possible that disease-related mutations can change the stability and flexibility of the coiled coil which can manifest itself as a specific phenotype.

The aim of this work was to find out whether different phenotypes, caused by sequences of muscle isoforms and disease-related missense mutations in TPM1 and TPM3, the genes encoding Tpm1.1 and Tpm3.12, can be associated with any particular structural changes in the mid-region of Tpm. To analyze local changes within period 3 and its vicinity, we constructed fragments of Tpm1.1 and Tpm3.12 containing the actin-binding period 3 and short sequences from periods 2 and 4 . The effects of four autosomal dominant missense mutations found in human TPM1 and TPM3 on the structural stability of these fragments were analyzed by circular dichroism (CD) and molecular dynamic simulation (MDS).

\section{Results}

\subsection{Design of Tpm1.1 and Tpm3.12 Peptides}

To limit the analyses to the conservative actin-binding period 3 and the sequences nearest to this period, we engineered 91-residue fragments of Tpm1.1 and Tpm3.12 comprising the entire 35-amino acid residue long actin-binding period 3 flanked by sequences of periods 2 and 4 at the $\mathrm{N}$ - and C-termini of period 3 (Figure 1). 
A

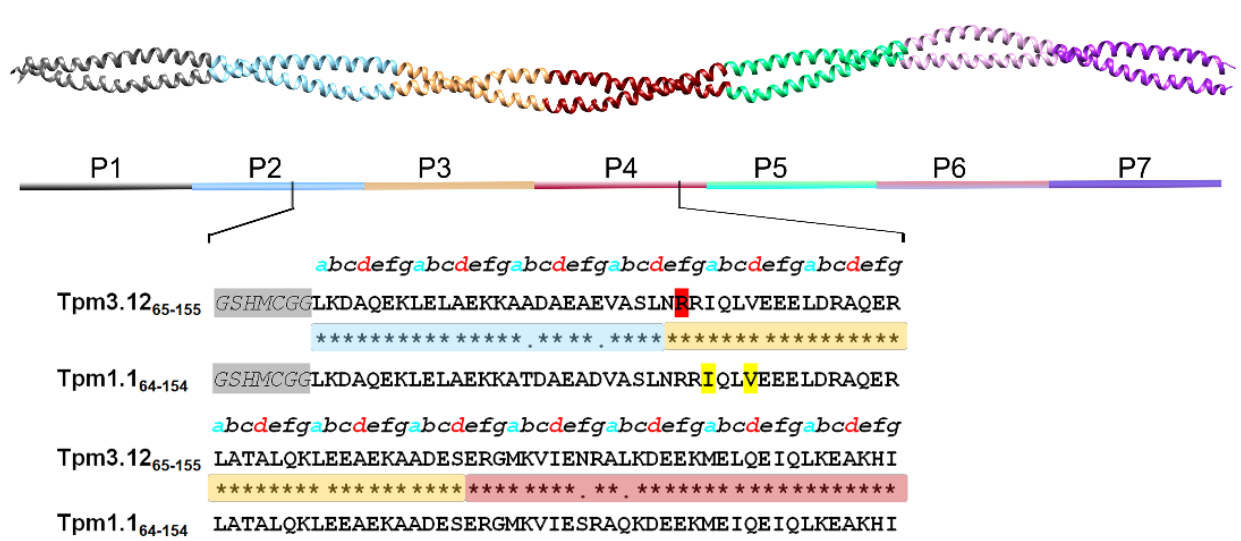

B

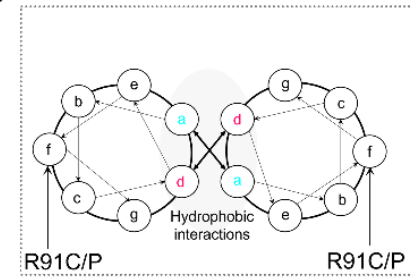

Tpm 3.12 mutants

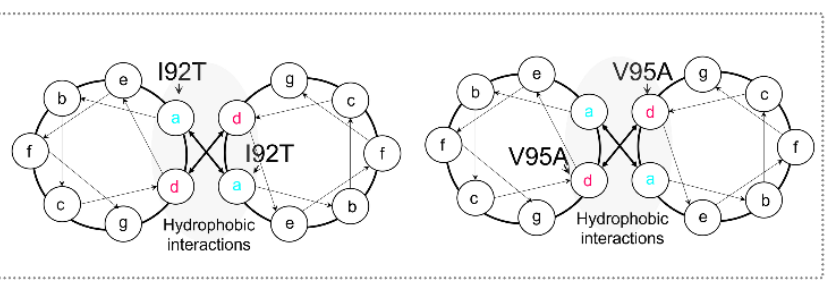

Tpm 1.1 mutants

Figure 1. Tpm1.1 and Tpm3.12 peptides design. (A) Schematic representation of the fragments with respect to the full-length model of Tpm (PDB ID: 1C1G). Sequence comparison of designed Tpm3.12 and Tpm1.1 fragments. The alignment was performed in UniProt, accession numbers P09493 for Tpm1.1 and P06753 for Tpm3.12. Stars between the sequences indicate identical amino acid residues, dots indicate sites where the sequences differ. Blue, orange, and magenta indicate positions of actin-binding periods 2, 3 and 4, respectively. Lower case italicized letters on top of the sequences show positions of the amino acid residues in heptad repeat and $a$ and $d$ positions are shown in cyan and red, respectively. Mutation sites related to hypo- and hypercontractile phenotypes are highlighted in yellow and red, respectively. The $\mathrm{N}$-terminal fusion peptide (shaded with gray) comprises amino acid residues left after proteolysis of His-tag with thrombin, followed by Cys and elastic Gly-Gly linker introduced during peptide construction. (B) Illustration of the coiled-coil wheel where positions of the mutations are indicated by arrowheads.

To ensure the undisturbed folding of the coiled coil, the sequence contained 13 heptad repeats. The Tpm1.1 peptide contained sequences from Leu64 to Ileu154 and was referred to as Tpm1.1 $164-154$. Since Tpm3.12 has an additional N-terminal Met, the amino acid residues in the sequence of Tpm3.12 have increased numbers compared to the sequence of Tpm1.1. For consistency with residue numbering in the full-length Tpm3.12, the peptide was designated as Tpm3.12 ${ }_{65-155}$. The sequences of the peptides differed in the following residues: Thr79/Ala80, Asp84/Glu85 (actin-binding period 2), Ser132/Asn133, and Gln135/Leu136 (actin-binding period 4). In addition, the peptide carried an N-terminal fusion sequence with Cys residue separated from the Tpm sequence by a flexible Gly-Gly linker and GSHM tetrapeptide left after His-tag proteolysis (see Materials and Methods). The presence of Cys residues allowed us to cross-link two Tpm chains to facilitate the formation of the coiled coil structure.

The selected disease-causing mutations were located in a short segment of actinbinding period 3 (Figure 1). The substitutions I92T and V95A in Tpm1.1 are known to cause dilated cardiomyopathy and hypertrophic cardiomyopathy characterized by hypo- and hypercontractile phenotypes, respectively [24-26]. Substitutions R91C and R91P in Tpm3.12 are causative of congenital myopathy with slow muscle fiber hypotrophy and muscle weakness [23,27]. The cardiomyopathy mutations I92T and V95A were located in the $a$ and $d$ positions of the heptad repeat, in the core of Tpm1.164-154 (Figures 1 and S1A). The con- 
genital myopathy substitutions R91C and R91P were in the $f$ position of the heptad repeat, which exposed them on the outer face of the Tpm3.12 ${ }_{65-155}$ coiled coil (Figures 1 and S1B).

\subsection{Structural Stability of Tpm1.1 $1_{64-154}$ and Tpm3.12 ${ }_{65-155}$}

Structural analyses were performed under reducing and non-reducing conditions. To confirm the formation of Tpm cross-links via S-S bond formation, we used SDS gel electrophoresis. As shown in Figure 2, boiling the peptides in the presence of $\beta$-mercaptoethanol reduced the disulfide bonds and separated both chains of the dimers. The wild-type peptides Tpm1.1 $1_{64-154}$ and Tpm3.12 ${ }_{65-155}$, migrated with the apparent molecular mass $\sim 11 \mathrm{kDa}$ (lanes 2 and 5), which roughly corresponded to the theoretical molecular mass of one peptide chain. In the absence of $\beta$-mercaptoethanol, mobility of both peptides corresponded to the molecular mass of dimers $\sim 22 \mathrm{kDa}$ (lanes 4 and 7). The dimeric form dominated even after boiling the samples in the absence of $\beta$-mercaptoethanol prior to the separation on the gels (lines 3 and 6).

During the thermal unfolding experiments, a protein undergoes a transition from a folded to unfolded state due to a gradual increase in temperature. Considering the unfolding process undergoes a two-state transition (folded-unfolded), the temperature at which the ratio of folded to unfolded equals 1 is regarded as the melting temperature $\left(T_{m}\right)$. As $T_{m}$ can be used to compare the structural stabilities of proteins with similar structures, the thermal unfolding experiments were performed under reducing and non-reducing conditions (Figure 3).

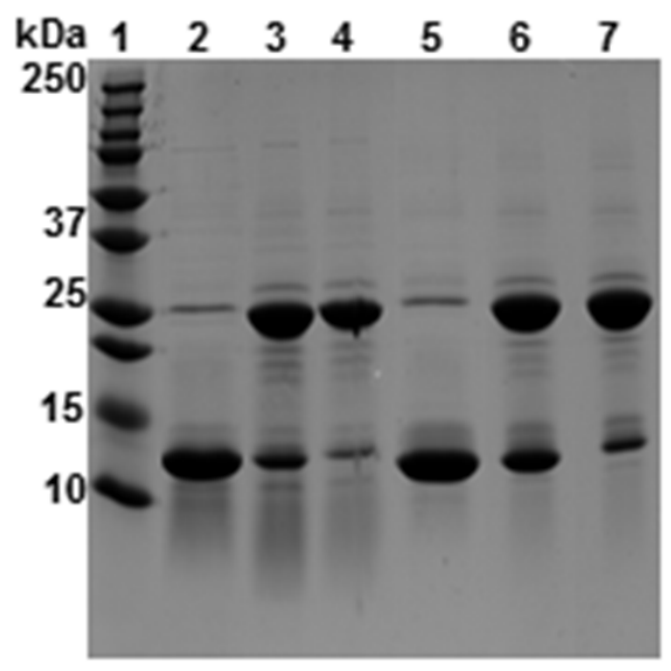

Figure 2. SDS-polyacrylamide gel electrophoresis of the wild-type peptides under reducing and non-reducing conditions. Tpm1.164-154 (lanes 2-4) and Tpm3.1265-155 (lanes 5-7) separated on the SDS-gels after incubation for $3 \mathrm{~min}$ at $100{ }^{\circ} \mathrm{C}$ with Laemmli solution in the presence of $10 \% \beta$-mercaptoethanol (lanes 2 and 5), in the absence of $\beta$-mercaptoethanol (lanes 3 and 6), or neither with boiling nor $\beta$-mercaptoethanol (lanes 4 and 7).

The values of $\mathrm{T}_{\mathrm{m}}$ computed from the melting curves are collected in Table 1. The Tpm3.12 ${ }_{65-155}$ cross-linked dimer displayed strong structural stability, which was approximately $15^{\circ} \mathrm{C}$ higher than the cross-linked Tpm $1.1_{64-154}$. Reducing the Cys residues with $0.5 \mathrm{mM}$ DTT caused a significant drop in the stability of both peptides, but similarly to the oxidized peptides, the reduced Tpm3.1265-155 was more stable than the reduced Tpm 1.1 $1_{64-154}$ (Table 1). While for Tpm3.1265-155 the difference in the $\mathrm{T}_{\mathrm{m}}$ values between nonreduced and reduced species was approximately $15.1^{\circ} \mathrm{C}$, Tpm $1.1_{64-154}$ showed $\sim 22.4^{\circ} \mathrm{C}$ difference between reduced and non-reduced species. 

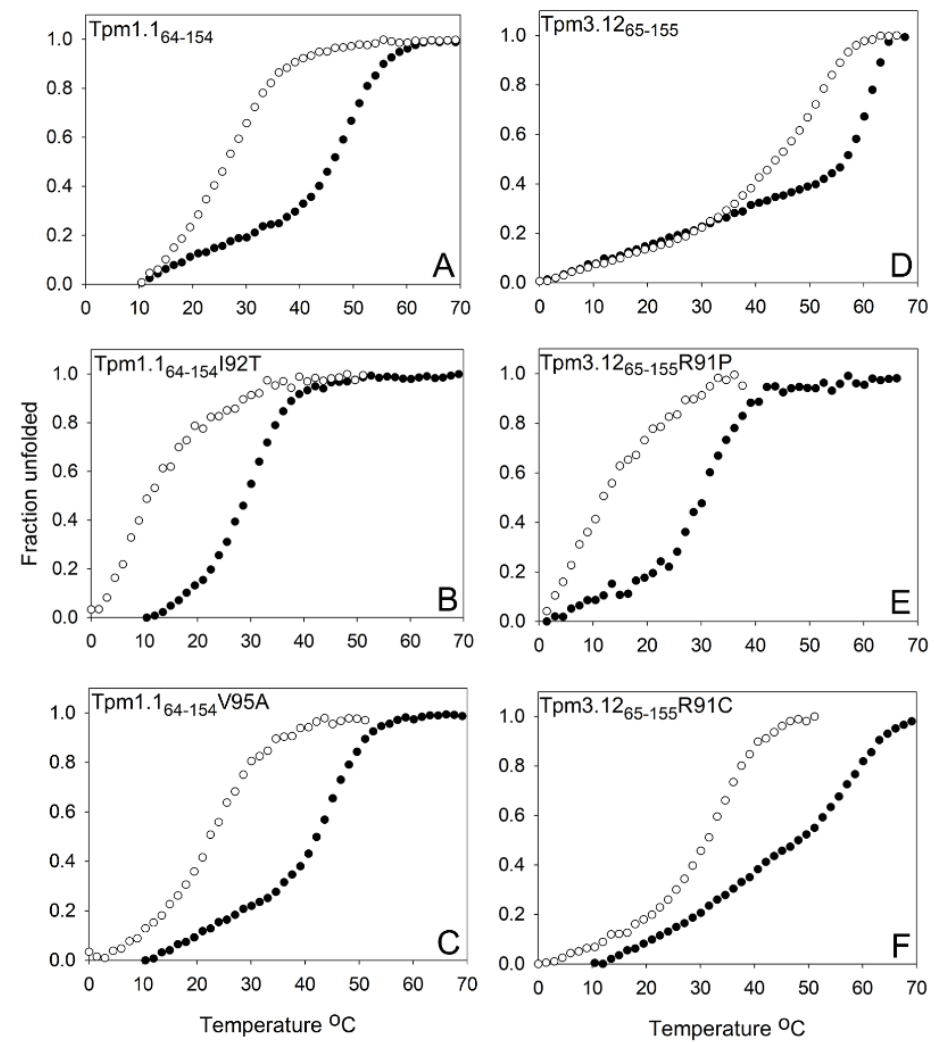

Figure 3. Effect of mutations on the thermal stability of Tpm1.1 $1_{64-154}$ and Tpm3.12 ${ }_{65-155}$. Melting of Tpm1.1 $1_{64-154}$ (A), Tpm3.12 $12_{65-155}(\mathbf{D})$, and their respective mutants $(\mathbf{B}, \mathbf{C}, \mathbf{E}, \mathbf{F})$ were measured at $222 \mathrm{~nm}$ in $100 \mathrm{mM} \mathrm{NaCl}, 10 \mathrm{mM} \mathrm{Na}$-Phosphate, $\mathrm{pH}$ 7.0, with $(\bigcirc)$ and without $(\bullet) 0.5 \mathrm{mM}$ DTT. Fraction unfolded is the ratio of unfolded to folded peptides (see Materials and Methods).

Table 1. Effect of the mutations on melting temperature $\left(T_{m}\right)$ of $T p m 1.1_{64-154}$ and $T p m 3.12_{65-155}$ under non-reducing and reducing conditions. Melting temperatures were determined from melting curves measured in $10 \mathrm{mM}$ Na-phosphate, $100 \mathrm{mM} \mathrm{NaCl}, \mathrm{pH} 7.0$, under reducing ( $0.5 \mathrm{mM} \mathrm{DTT}$ ) and non-reducing (no DTT) conditions. ND: not determined. The numbers are mean values from three measurements \pm SE.

\begin{tabular}{|c|c|c|}
\hline \multirow[t]{2}{*}{ Tpm } & \multicolumn{2}{|c|}{ Melting Temperature $\left(\mathrm{T}_{\mathrm{m}}\right),{ }^{\circ} \mathrm{C}$} \\
\hline & No DTT & DTT \\
\hline Tpm1.164-154 & $46.1 \pm 0.2$ & $23.7 \pm 0.7$ \\
\hline Tpm1.1 $164-154 \mathrm{I} 92 \mathrm{~T}$ & $28.2 \pm 0.1$ & $5.6 \pm 0.5$ \\
\hline Tpm1.1 $64-154$ V95A & $42.2 \pm 0.5$ & $21.7 \pm 0.3$ \\
\hline Tpm 3.12 $65-155$ & $60.9 \pm 0.2$ & $45.8 \pm 0.9$ \\
\hline Tpm 3.12 $65-155$ R91C & $51.5 \pm 0.7$ & $31.3 \pm 0.2$ \\
\hline Tpm3.1265-155R91P & $28.9 \pm 0.1$ & ND \\
\hline
\end{tabular}

\subsection{Disruptive Effects of Missense Mutations on Stability of Tpm1.164-154 and Tpm3.1265-155}

In Tpm1.1 $1_{64-154}$, the I92T mutation appeared to be more disruptive than the V95A mutation (Table 1, Figure 3). Under both reducing and non-reducing conditions, the I92T mutation resulted in a decrease in $\mathrm{T}_{\mathrm{m}}$ by $\sim 18{ }^{\circ} \mathrm{C}$, and the decrease caused by the V95A mutation was only 3.9 and $2.0^{\circ} \mathrm{C}$ for non-reduced and reduced peptides, respectively.

A decrease in stability was also observed in Tpm3.12 $65-155$ when R91 was changed either to Cys or Pro. Under both non-reducing and reducing conditions, Tpm3.12 ${ }_{65-155} \mathrm{R} 91 \mathrm{P}$ was less stable than Tpm3.12 ${ }_{65-155} \mathrm{R} 91 \mathrm{C}$. While the $\mathrm{R} 91 \mathrm{C}$ mutation caused a $\sim 9.4^{\circ} \mathrm{C}$ decrease in $\mathrm{T}_{\mathrm{m}}$, the R91P mutation resulted in a $32{ }^{\circ} \mathrm{C}$ decrease in $\mathrm{T}_{\mathrm{m}}$ indicating a drastic decrease in thermal stability of the cross-linked Tpm3.12 ${ }_{65-155}$. Under reducing conditions, $\mathrm{T}_{\mathrm{m}}$ 
for Tpm3.12 $25-155 \mathrm{R} 91 \mathrm{P}$ cannot be determined due to the lack of the beginning phase of the two-state transition (Figure 3E). In contrast, Tpm3.12 ${ }_{65-155} \mathrm{R} 91 \mathrm{C}$ showed the two-state transition (Figure 3F), as compared to Tpm3.1265-155, its $\mathrm{T}_{\mathrm{m}}$ was decreased by $\sim 14.5^{\circ} \mathrm{C}$.

\subsection{R91P Mutation Increased Disorder in Tpm3.12 $65-155$}

In order to further evaluate the folding of Tpm3.12 ${ }_{65-155} \mathrm{R} 91 \mathrm{P}$ under reducing conditions, $\mathrm{CD}$ spectra were measured to evaluate the secondary structure changes induced by the R91P mutation (Figure 4). Under non-reducing conditions, Tpm3.12 ${ }_{65-155}$ R91P showed a profile characteristic for a protein that is mostly $\alpha$-helical; however, upon treatment with DTT, the structure became significantly disordered as judged by the shift of the X-axis intersection point to lower wavelengths and the increase in the mean residue ellipticity at $222 \mathrm{~nm}$ from $-28,370$ to $-19,057$ deg.cm ${ }^{2} . \mathrm{dmol}^{-1}$, which accounts for the $\sim 40 \%$ decrease in $\alpha$-helical content.

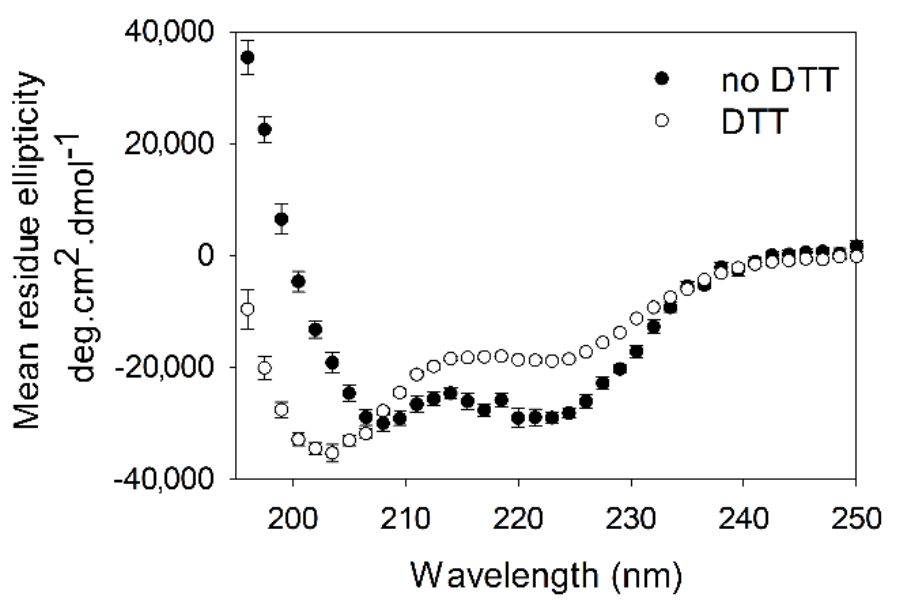

Figure 4. The R91P substitution in Tpm3.12 $65-155$ results in decreased helicity in the presence of DTT. $\mathrm{CD}$ spectra were measured at $0{ }^{\circ} \mathrm{C}$ in $100 \mathrm{mM} \mathrm{NaCl}, 10 \mathrm{mM}$ Na-Phosphate, $\mathrm{pH}$ 7.0, with or without $0.5 \mathrm{mM}$ DTT.

\subsection{Analysis of Three-Dimensional Structures of the Tpm Fragments Using Molecular Dynamics Simulations}

No high-resolution 3D structures for Tpm1.1 and Tpm3.12 are available; therefore, we used a low-resolution (7 $\AA$ ) X-ray crystal structure of cardiac $\alpha$-tropomyosin from Sus scrofa (PDB ID 1C1G) as a template to create PDB files for Tpm1.1 $1_{64-154}$ and Tpm3.12 ${ }_{65-155}$. A Tpm fragment is an elongated molecule; therefore, end-to-end distance enables us to assess the bending propensity of this molecule. $\mathrm{N}$ and $\mathrm{C}$ termini of the designed fragments were disordered. To exclude the disordered regions and consider only the coiled-coil part of the Tpm fragment, $\mathrm{C} \alpha$ distances between the first and last residues forming the $\alpha$-helix were measured over the course of the simulation. The $\mathrm{C} \alpha-\mathrm{C} \alpha$ distances $(\AA)$ between L64-K152 in Tpm1.1 $1_{64-154}$ (both chains), L65-E151, and L65-K153 in Tpm3.1265-155 (chains A and $\mathrm{B}$, respectively), and their respective mutant fragments were measured and plotted as a function of time during the $200 \mathrm{~ns}$ simulation run (Figure S2, Table S1).

In Tpm1.1 $1_{64-154}$, the range of end-to-end distance fluctuations was 55-95 $\AA$ (Figure S2). In Tpm3.12 $265-155$, the range of fluctuations was 55-100 $\AA$, which was similar to Tpm1.1 $164-154$. Interestingly, the distances for the chains $A$ and $B$ in dimers fluctuated more independently in Tpm1.164-154. Over the time of the simulation run, the distances were $78.24 \pm 6.23 \AA$ and $73.43 \pm 5.74 \AA$ for chains A and B, respectively (Table S1). In contrast, chains A and $B$ of Tpm3.12 $12_{65-155}$ maintained similar end-to-end distances with respect to each other $(78.9 \pm 9.3 \AA$ and $76.7 \AA \pm 11.1 \AA$, respectively). The average distances for the fragments calculated as an average of the end-to-end distances of both $\mathrm{A}$ and $\mathrm{B}$ chains were $75.8 \pm 4.9 \AA$ for Tpm $1.1_{64-154}$ and $77.78 \AA \pm 9.9 \AA$ for Tpm 3.1265-155. The differences in 
the average end-to-end distances for the individual chains can be attributed to the changes in the conformation of terminal residues (helical vs. extended).

We expected that introducing mutations in the pdb files of the fragments and running simulations would result in the disruption of the coiled-coil structures. Surprisingly, $200 \mathrm{~ns}$ simulation runs were not enough to show any effects on distances between individual chains of coiled coil. For all mutations besides I92T, there were also no significant changes in the end-to-end distances (Figure S2, Table S1). When the substitution I92T was introduced in Tpm1.1 $164-154$, we observed fluctuations occurring between 40 and $80 \AA$, with an average distance $58.4 \pm 7.2 \AA$.

To test if the distances measured during simulations were dependent on the positions of the side chains in the starting structures created in Chimera, we changed the orientation of the side chains of several residues that form intrachain salt bridges so that the salt bridges were broken. New chosen positions were also allowable and highly probable according to [28]. Then, simulations were repeated for Tpm1.1 $164-154$ and Tpm1.164-154 $192 \mathrm{~T}$ (Figure S3), and the average distances became $68.0 \pm 6.8$ and $75.73 \pm 9.6$, respectively. Based on this result, we concluded that the end-to-end distance was not a reliable parameter to determine the flexibility of coiled-coil structures created in silico.

\section{Discussion}

The flexibility of the Tpm coiled coil was reported to be crucial for the binding of Tpm to F-actin and the regulation of actomyosin interactions (reviewed by [1]). The highly conservative middle region comprising actin-binding period 3 is particularly important for the cooperativity of the actin filament regulation $[20,21]$. In humans, mutations in Tpm genes encoding actin-binding period 3 were linked to congenital myopathies and cardiomyopathies, muscle diseases that are manifested either by hypo- or hypercontractile phenotypes. In different Tpm genes, disease-causing mutations do not overlap, indicating that some regulatory properties are isoform-specific. It is not clear whether any particular structural changes in Tpm can be associated with Tpm functions leading to these phenotypes. To address this problem, in this work, we investigated the structural stability of peptides equivalent to sequences of the middle regions of Tpm1.1 and Tpm3.12, Tpm isoforms expressed in fast and slow muscle fibers, which contained sequences of actin-binding period 3 flanked by sequences from periods 2 and 4 . The stabilities of wild-type peptides were compared to the stabilities of peptides carrying disease-causing missense mutations in period 3, which cause hypo- and hypercontractile phenotypes. CD measurements of thermal stability revealed that the middle region of Tpm3.12 was more stable than Tpm1.1. The mutations decreased thermal stability in the peptides with different degrees and had various effects on the peptide structure, but we did not observe any particular structural changes which determined the opposite phenotypes.

\subsection{Differences in Stability of Tpm1.1 and Tpm3.12 Middle Regions}

Thermodynamic studies of the stability of full-length Tpm1.1 and Tpm3.12 revealed the presence of three calorimetric domains in both isoforms, one of which was assigned to the N-terminal half of the molecule; for Tpm3.12, the thermal transition of the N-terminal domain was approximately $7{ }^{\circ} \mathrm{C}$ higher than for Tpm1.1 [29]. Although the peptides Tpm1.1 $164-154$ and Tpm3.1265-155, which were studied in this work, contained only part of the $N$-terminal domain, they showed an even bigger, $\sim 15^{\circ} \mathrm{C}$, difference in the thermal transition from fully folded to unfolded conformation. Tpm1.1 $1_{64-154}$ was less stable than Tpm3.1265-155. The lower stability can be ascribed to the four amino acid differences between the peptides (Figure 1). It is interesting that the isoform-specific amino acid residues are located in the $b, g$ and $f$ positions of the heptad repeat. The $g$ position amino acid residue, which electrostatically stabilizes the coiled coil, is a conservative Asp/Glu substitution; therefore, one can suppose that the high stability of Tpm3.12 mid-region is maintained by long-range conformational changes. 
Because a certain degree of flexibility positively affects the binding of Tpm to actin, the difference between Tpm1.1 $1_{64-154}$ and Tpm3.12 $65-155$ observed in these studies may explain a higher affinity of Tpm3.12 for actin [5,6]. In agreement with the stabilizing effect of single Cys oxidation on the full-length Tpms [29], cross-linking of the peptide chains by oxidation of the N-terminal Cys increased $\mathrm{T}_{\mathrm{m}}$. Together, the results obtained in this work indicate that the middle region extending from Leu64(65) to Ser154(155) significantly contributes to the conformation and stability of Tpm1.1 and Tpm3.12.

\subsection{Effects of Disease-Causing Mutations on Structural Transitions of Tpm1.1 and Tpm3.12 Middle Regions}

From the CD experiments, drastic decreases in the stability of the designed Tpm peptides in the presence of DTT indicate that the SS bond formed by cysteines in the Nterminal sequence GSHMCGG was effective in stabilizing Tpm's coiled-coil structure. This addition to the $\mathrm{N}$-terminal sequence could serve as an effective strategy in assessing the structural and functional relevance of Tpm's periodicity in actin regulation and effects of other disease-related mutations in Tpms. The SS bond formed by the introduced N-terminal Cys mimics the stable N-terminal coiled coil that would precede this region in full-length Tpm and allows to ensure the formation of a coiled coil by the peptide. Introducing an additional C-terminal Cys that will form one more SS bond is not necessary for the coiled coil formation; furthermore, it may introduce an unnecessary limitation in observing the downstream structural effects of the mutations in the coiled coil. Due to the SS bond formation, our fragments have increased stability. For Tpm3.12 $65-155, \mathrm{~T}_{\mathrm{m}}$ is $61^{\circ} \mathrm{C}$ (crosslinked), while $\mathrm{T}_{\mathrm{m}}$ of the corresponding region in full-length Tpm3.12 (Domain 3) is $\sim 57^{\circ} \mathrm{C}$ (reduced) or $\sim 58{ }^{\circ} \mathrm{C}$ (cross-linked) [29,30]. For Tpm1.164-15, Tm is $46{ }^{\circ} \mathrm{C}$ (cross-linked), while $\mathrm{T}_{\mathrm{m}}$ values of domains of full-length $\mathrm{Tpm} 1.1$ vary from 38 to $51{ }^{\circ} \mathrm{C}$ (reduced) [31]. Therefore, our designed cross-linked fragments have stability close to full-length Tpm.

As shown in Figure 1, Tpm 1.1 mutations I92T and V95A are in the core of the coiled coil ( $a$ and $d$ positions). These mutations were shown to be associated with DCM and HCM respectively [24], indicating opposite effects of these mutations on the functions of Tpm. Tpm1.164-154 V95A displayed structural stability similar to that of wild-type Tpm1.1 $1_{64-154}$ as indicated by $\sim 4{ }^{\circ} \mathrm{C}$ and $\sim 2{ }^{\circ} \mathrm{C}$ decreases in the presence and absence of DTT (Table 1). Melting experiments indicated that Tpm1.164-154 V95A performed better in the absence of DTT in retaining the coiled coil structure. In contrast to V95A, I92T mutation in Tpm1.164-154 resulted in a significant decrease in the coiled coil stability, as suggested by the drop in $\mathrm{T}_{\mathrm{m}}$ of $\sim 18^{\circ} \mathrm{C}$ in the presence and absence of DTT.

R91 in Tpm 3.12 makes an electrostatic interaction with actin, and it has been previously shown that substitution of R91 with Cys reduced Tpm's actin affinity, thereby affecting F-actin pointed end regulation [23]. Based on our CD data, introducing the R91C mutation resulted in $\sim 9{ }^{\circ} \mathrm{C}$ and $\sim 15{ }^{\circ} \mathrm{C}$ decreases $\mathrm{T}_{\mathrm{m}}$ in the absence and the presence of DTT, respectively (Table 1). If an SS bond can be formed by C91, we would expect an increase in stability. However, side chains of cysteine residues at the 91st position are located on the opposite sides of the coiled coil and cannot form the SS bond (Figure S1). Thus, the difference between $\mathrm{T}_{\mathrm{m}}$ in the presence and the absence of DTT can be attributed to the disulfide bond formed by Cys in the N-termini of the designed fragments and not by C91. A disrupted coiled coil in Tpm3.12 ${ }_{65-155}$ R91C contributes to the reduced affinity of Tpm for actin shown previously [32].

Studies on the thermostability of the full-length Tpm3.12-R91P showed that the substitution caused a conformational change, which resulted in a loss of the N-terminal calorimetric domain [30]. In this work, we showed that the substitution severely affected the region near the substitution. Given the destructive effect of Pro on the $\alpha$-helix formation [33], the significant loss of the $\alpha$-helical structure resulting in a dramatic loss of stability of Tpm3.12 ${ }_{65-155} \mathrm{R} 91 \mathrm{P}$ is not surprising. Along similar lines, the R91P mutation instigated the disruption of coiled-coil structure as shown by the difference of $32{ }^{\circ} \mathrm{C}$ in $\mathrm{T}_{\mathrm{m}}$ in the absence of DTT. $\mathrm{T}_{\mathrm{m}}$ could not be determined in the presence of DTT as the Tpm3.12 ${ }_{65-155} \mathrm{R} 91 \mathrm{P}$ had already begun unfolding at $0{ }^{\circ} \mathrm{C}$ (Table 1 , Figure 3$)$. The presence of DTT prevented 
the formation of an SS bond at the N-terminal sequence, thereby attributing to the drastic difference in the stability of the Tpm3.12 ${ }_{65-155} \mathrm{R} 91 \mathrm{P}$ fragment. Tpm3.12 ${ }_{65-155} \mathrm{R} 91 \mathrm{P}$ loses its coiled-coil structure significantly as indicated by the increase in the disorder and loss of helical content (Figure 4).

Surprisingly, in the 200 ns MDS run, the substitution of R91 with P showed no significant changes in the coiled-coil structure of Tpm3.1265-155 R91P. Conventional MDS for time frames of $200 \mathrm{~ns}$ and similar have been useful in determining the effects of mutations on protein structural behavior and protein-protein interactions [34]. However, it still remains a challenge to show an experimentally-determined disruptive and slow dissociation of protein-protein interactions due to time frames ranging from micro to milliseconds or even longer $[35,36]$. Due to the limitations of conventional MDS and the use of shorter timeframes, specific disruptive effects of the Tpm3.12 ${ }_{65-155} \mathrm{R} 91 \mathrm{P}$ and Tpm1.1 ${ }_{65-155} \mathrm{I} 92 \mathrm{~T}$ mutation on coiled coil destabilization seen in the CD experiments remain elusive.

The mutations $192 \mathrm{~T}$ and $\mathrm{R} 91 \mathrm{P}$ result in the loss of the local coiled-coil structure in the region of the actin-binding period 3. At the body temperature $\left(\sim 37^{\circ} \mathrm{C}\right)$, this region becomes unfolded; this should drastically affect the binding of Tpm to F-actin. Two other mutations, V95A and R91C, cause partial unfolding in this region at the body temperature, the interactions between Tpm and F-actin should also be affected, although to a lesser extent. Moreover, Tpm has a multidomain structure with several cooperatively melting domains, and the unfolding of one domain has allosteric effects on the stability of the subsequent domains [37].

\section{Materials and Methods}

\subsection{Construction and Cloning of TPM1 and TPM3 cDNA Fragments}

cDNA encoding fragments of Tpm1.1 and Tpm3.12 comprising amino acid residues 64-154 or 65-155 were excised from TPM1 and TPM3 cDNAs encoding human skeletal tropomyosin isoforms using PCR amplification.

The TPM1 cDNA sequence was amplified by PCR with forward:

5'TAGTACATATGTGCGGCGGCCTCAAAGATGCCCAGGAG3' and reverse 5'TAGATGGATCCTTAAATGTGCTTGGCCTCTTTC3' primers.

The TPM3 cDNA sequence was amplified with forward:

5'TAGTACATATGTGCGGCGGCCTGAAAGACGCTCAGGAAAAACTG $3^{\prime}$ and reverse 5'TAGATGGATCCTTAGATGTGTTTAGCTTCTTTCAGC3' primers. The NdeI restriction site and the sequence encoding CysGlyGly upstream of the sequences encoding Tpm1.1 and Tpm3.12 were added to the $5^{\prime}$-end of the fragments. A BamHI restriction site and a TAA translation termination codon were introduced at the $3^{\prime}$-end of the amplified fragments. DNA fragments were amplified by PCR using Taq DNA polymerase (New England BioLabs, Inc., Ipswich, MA, USA) and the appropriate PCR buffer at $10 \times$ concentration with $17.5 \mathrm{mM} \mathrm{MgCl}$. The reaction mixture $(50 \mu \mathrm{L})$ contained $0.15 \mathrm{mM} \mathrm{dNTPs,}$ $1 \times$ Taq DNA polymerase buffer, 30 pmoles of each primer, $1 \mathrm{U}$ polymerase, and $80 \mathrm{ng}$ of template plasmid. The first amplification was preceded by 4 min incubation at $94{ }^{\circ} \mathrm{C}$ and then DNA polymerase was added. The amplification parameters were as follows: denaturation at $94{ }^{\circ} \mathrm{C}$ for $30 \mathrm{~s}$ and extension at $72{ }^{\circ} \mathrm{C}$ for $25 \mathrm{~s}$. Annealing was conducted for $15 \mathrm{~s}$ at $48^{\circ} \mathrm{C}$ for TPM1 and at $50.5^{\circ} \mathrm{C}$ for TPM3. The number of amplification cycles was set to 20 . The procedure was completed by 5 min incubation at $72^{\circ} \mathrm{C}$. The PCR fragments were digested with $\mathrm{NdeI}$ and BamHI. The obtained sequences were placed under the transcriptional control of the T7 promoter of T7 bacteriophage in the pET15b expression vector. For this purpose, TPM1 and TPM3 sequences coding amino acid residues 64-154 and 65-165 were cloned into the pET15b backbone between the NdeI and BamHI restriction sites. The plasmids were amplified in the $\mathrm{DH} 5 \alpha$ strain (Stratagene, La Jolla, CA USA) according to the method of the manufacturer. The validity of the cDNA sequences introduced into the pET15b plasmid was confirmed by DNA sequencing. 


\subsection{Site-Directed Mutagenesis}

Fragments of TPM1 and TPM3 cloned into the pET15b vector were used as templates. Cardiomyopathy-related mutations I92T and V95A were introduced into the TPM1 cDNA. CFTD-causing mutations R91C and R91P were introduced into the TPM3. The mutations were inserted using a PCR-based oligonucleotide-directed mutagenesis kit (Agilent Technologies, Santa Clara, CA, USA). The following oligonucleotides were used:

I92T: forward 5'CATCTCTGAACAGACGCACCCAGCTGGTTGAGG3'

and reverse $3^{\prime}$ GTAGAGACTTGTCTGCGTGGGTCGACCAACTCC5';

V95A: forward 5'ACGCATCCAGCTGGCTGAGGAGGAGTTGG3'

and reverse $3^{\prime}$ TGCGTAGGTCGACCGACTCCTTCAACC5'

R91C: forward 5'GAAGTTGCTTCTCTGAACTGTCGTATCCAGCTGGTTG3'

and reverse $3^{\prime}$ CTTCAACGAAGAGACTTGACAGCATAGGTCGACCAAC5';

R91P: forward 5'GAAGTTGCTTCTCTGAACCCTCGTATCCAGCTGGTTG3'

and reverse $3^{\prime}$ CTTCAACGAAGAGACTTGGGAGCATAGGTCGACCAAC5' .

The changed codons are underlined. The oligonucleotides were synthesized and

HPLC was purified by the Laboratory of DNA Sequencing and Oligonucleotide Synthesis, Institute of Biochemistry and Biophysics (Warsaw, Poland). The PCR reaction was performed according to the method supplied by the manufacturer (https: / / www.agilent. $\mathrm{com} / \mathrm{cs} /$ library/usermanuals/public/200523.pdf, accessed on 15 November 2021). The amplification reaction involved initial DNA melting $\left(95^{\circ} \mathrm{C}, 30 \mathrm{~s}\right)$, then 11 cycles of melting $\left(95^{\circ} \mathrm{C}, 30 \mathrm{~s}\right)$, and primers annealing $\left(55^{\circ} \mathrm{C}, 1 \mathrm{~min}\right)$ and extending $\left(68^{\circ} \mathrm{C}, 6 \mathrm{~min}\right)$. The parental DNA template was digested with the DpnI restriction enzyme and PCR products were transformed into XL-1 supercompetent cells, DNA was isolated (GeneMATRIX Plasmid Miniprep DNA Purification Kit, EURx), and the substitutions were verified by DNA sequencing in the Laboratory mentioned above.

\subsection{Peptide Expression and Purification}

Wild-type and mutant Tpm1.1 $1_{64-154}$ and Tpm3.12 $65-155$ were expressed in E.coli BL21 (DE3) competent cells (Novagen Inc. Madison, WI, USA). The cells were transformed with wild-type and mutant plasmids TPM1 and TPM3 by 30-sec heat shock at $42{ }^{\circ} \mathrm{C}$ according to the protocol of the manufacturer. Transformed cells were grown overnight in Luria-Bertani (LB) broth supplemented with $0.05 \mathrm{mg} / \mathrm{mL}$ ampicillin, at $37^{\circ} \mathrm{C}$, without shaking. Peptide production was induced by $0.4 \mathrm{mM}$ IPTG at culture $\mathrm{OD}_{550}$ between 0.2 and 0.4 . The culture was grown with shaking $270 \mathrm{rpm}$ at $37^{\circ} \mathrm{C}$ until $\mathrm{OD}_{550}$ reached 1.0. Cells were pelleted by $10 \mathrm{~min}$ centrifugation in a GSA Sorval rotor at $11,000 \mathrm{rpm}, 4^{\circ} \mathrm{C}$. To release the peptides, pellets collected from $600 \mathrm{~mL}$ of culture were suspended in $12 \mathrm{~mL}$ of lysis buffer $(50 \mathrm{mM}$ Tris- $\mathrm{HCl}$, pH 7.5, $10 \mathrm{mM}$ EDTA, $20 \%$ saccharose, $0.5 \mathrm{mg} / \mathrm{mL}$ lysozyme), homogenized in Dounce homogenizer, incubated for $1 \mathrm{~h}$ on ice, and then for $10 \mathrm{~min}$ at $-80^{\circ} \mathrm{C}$. After thawing, $\mathrm{NaCl}$ was added to a $0.2 \mathrm{M}$ final concentration, and the extract was frozen for $10 \mathrm{~min}$ at $-80^{\circ} \mathrm{C}$. Finally, $\mathrm{NaCl}$ concentration was increased to $1 \mathrm{M}$, and the solution was subject to two cycles of sonication using the Sonicator (Bandelin Sonoplus HD2070-BANDELIN electronic GmBH \& Co. Berlin, Germany) for $3 \mathrm{~min}$ at $70 \%$ maximal power. Cell debris was pelleted in Beckman rotor 70Ti for $1 \mathrm{~h}$, at 40,000 rpm, $4{ }^{\circ} \mathrm{C}$. Supernatants were collected and incubated in a water bath at $100{ }^{\circ} \mathrm{C}$ for $4 \mathrm{~min}$. The denatured proteins were pelleted in a 70Ti rotor, at $30,000 \mathrm{rpm}$, at $4{ }^{\circ} \mathrm{C}$ for $30 \mathrm{~min}$. Supernatants were dialyzed overnight to Abuffer (50 mM phosphate buffer, $\mathrm{pH} 8.0,0.3 \mathrm{M} \mathrm{NaCl}, 10 \mathrm{mM}$ imidazole, $0.02 \% \mathrm{NaN}_{3}$ ). The peptides were purified by affinity chromatography. The protein solution was loaded on HisSelect Nickel Affinity Resin (Sigma-Aldrich Corp. St Louis, MO, USA) column equilibrated with A-buffer and eluted with B-buffer (50 mM phosphate buffer $\mathrm{pH} 8.0,0.3 \mathrm{M} \mathrm{NaCl}$, $250 \mathrm{mM}$ imidazole, $0.02 \% \mathrm{NaN}_{3}$ ). Fractions from $2 / 3$ of the elution peak were collected and dialyzed exhaustively against $20 \mathrm{mM}$ Tris $\mathrm{pH} 7.5,0.1 \mathrm{M} \mathrm{NaCl}, 10 \mathrm{mM}$ EDTA, $0.02 \% \mathrm{NaN}_{3}$. His-tag was removed from the peptides by the Thrombin Cleavage Capture Kit (Novagen Inc., Madison, WI, USA). His-tagged Tpm peptides were incubated overnight at room temperature with biotinylated thrombin ( $0.02 \mathrm{U} / 1 \mathrm{mg}$ of protein). After digestion, thrombin 
was removed by incubation for $30 \mathrm{~min}$ at room temperature with streptavidin-agarose resin, followed by centrifugation in filter columns. Protein concentration was measured with the micro-biuret method [38]. Peptides were dialyzed to $50 \mathrm{mM}\left(\mathrm{NH}_{4}\right) \mathrm{HCO}_{3}$, lyophilized, and stored at $-80^{\circ} \mathrm{C}$. The identity of the peptides was confirmed by mass spectrometry (Liquid Chromatography and Mass Spectrometry Laboratory, Faculty of Biology, University of Warsaw, Poland). The obtained molecular weights (MW) corresponded to the theoretical MWs of dimers with oxidized Cys residues calculated in Expasy (https: / web.expasy.org / compute_pi/, accessed on 15 November 2021) based on the sequences of the peptides.

\subsection{Peptide Concentration Determination}

Protein concentration was measured with the micro-biuret method [38] or/and with the Pierce ${ }^{\mathrm{TM}}$ BCA protein assay kit (Thermo Fisher Scientific, Waltham, MA, USA). BCA uses the combination of the biuret reaction and the colorimetric detection of cuprous cations $\left(\mathrm{Cu}^{+}\right)$, which are influenced by the presence of copper-reducing amino acids cysteine, tyrosine, and tryptophan. Due to the lack of tyrosine and tryptophan residues in the Tpm fragments, other standard methods for protein concentration determination could not be used.

\subsection{Circular Dichroism Measurements}

Samples for CD experiments were dissolved in $10 \mathrm{mM}$ sodium phosphate, $100 \mathrm{mM}$ $\mathrm{NaCl}, \mathrm{pH} 7.0$ to a final concentration of $\sim 0.1 \mathrm{mg} / \mathrm{mL}$. Tpm3.12 ${ }_{65-155}$ and Tpm3.12 ${ }_{65-155} \mathrm{R} 91 \mathrm{P}$ fragments were resuspended in appropriate volumes of $100 \mathrm{mM}$ Tris pH 8.0, 0.1 M PMSF. The samples were acidified to a final concentration of $0.2 \mathrm{M} \mathrm{HCl}$ using $1 \mathrm{M} \mathrm{HCl}$. The samples were purified using a Sep-Pak C18 cartridge (Waters, Milford, CA, USA) and were eluted using a solution of $0.1 \%$ trifluoroacetic acid (TFA) containing $60 \%$ acetonitrile. The eluted samples were lyophilized to get rid of acetonitrile. Any impurities, if present, were removed by the Vydac 218TP C18 HPLC column using a linear gradient of acetonitrile, $1 \%$ per minute, in the presence of $0.1 \%$ TFA. An HPLC fraction containing the majority of the Tpm3.12 peak was collected in a glass vial and lyophilized. No additional peaks were found during HPLC, indicating the absence of any significant impurities. Lyophilized fractions were pooled and resuspended using minimal volumes of autoclaved HPLC water. The pooled fractions were lyophilized.

After the lyophilized Tpm fragments were resuspended, we noticed that not all fragments were soluble. In order to improve the solubility of the fragments, we refolded them by dialysis in the presence of urea. Tpm3.12 $65-155$ was resuspended in $20 \mathrm{mM}$ Tris $\mathrm{pH}$ 8.0, $8 \mathrm{M}$ urea, and $100 \mathrm{mM}$ DTT. The resuspended samples were dialyzed against $20 \mathrm{mM}$ Tris $\mathrm{pH} 8.0$ with concentrations of urea gradually reduced to $0 \mathrm{M}$ in the following order: 8 M-6 M-5 M-4 M-3.5 M-3 M-2.5 M-2.0 M-1.5 M-1 M-0.5 M-0 M, with a 12h duration between each step change. At $4 \mathrm{M}$ urea, the concentration of DTT was changed to $1 \mathrm{mM}$ and $100 \mathrm{mM} \mathrm{NaCl}$ was introduced into the dialysis buffer. Dialyzed soluble Tpm3.1265-155 were stored at $-80{ }^{\circ} \mathrm{C}$ until further use.

CD spectra measurements were performed using an Aviv model 400 spectropolarimeter (Lakewood, NJ, USA) in $1 \mathrm{~mm}$ quartz cuvettes. Thermal denaturation measurements were obtained by monitoring the ellipticity signal at $222 \mathrm{~nm}$ as a function of temperature. For each unfolding curve, a melting temperature $\left(\mathrm{T}_{\mathrm{m}}\right)$ was determined as a maximum of the first derivative using the Aviv instrument control program-Aviv 420 cdsxm (Lakewood, NJ, USA). Melting data for Tpm fragments were normalized using the following equation:

$$
z_{i}=\frac{x_{i}-\min (x)}{\max (x)-\min (x)}
$$

where, $x_{i}$ represents the $C D$ signal at a given temperature, $\min (x)$ represents the lowest CD signal, and $\max (\mathrm{x})$ represents the highest CD signal where there is maximal folding. Thermal denaturation profiles were normalized and plotted against temperature using SigmaPlot 12 (Systat Software, San Jose, CA, USA). 
CD spectra for the purified fragments, additionally purified using HPLC, were compared with fragments without the additional purification and no difference in spectra was observed. Therefore, purification and refolding procedures were not repeated for other Tpm fragments.

\subsection{Molecular Dynamics Simulations}

Protein structure building, editing, and visualization were performed with UCSF Chimera [39]. To build a starting structure for Tpm1.1 $1_{64-154}$, the X-ray crystal structure of cardiac $\alpha$-tropomyosin from Sus scrofa (PDB ID 1C1G) was used as a template to create PDB files for Tpm1.1 $1_{64-154}$ and Tpm3.1265-155. The crystal structure was edited by deleting M1 to A64 and A155 to I284 amino acid residues from chains B and D, and the remaining residues were changed according to the Tpm1.1 sequence (res. 64-154). The sequence GSHMCGG was added to the N-terminus of the fragment. A starting structure for Tpm3.12 ${ }_{65-155}$ was created by changing four residues in the Tpm1.1 $1_{64-154}$ structure.

Structures were neutralized with $\mathrm{Na}^{+}$or $\mathrm{Cl}^{-}$ions and solvated in TIP3P water [40] with at least $10 \AA$ between the structure and the edge of the solvation box. Energy minimizations were performed by the sander protocol in Amber18 [41] with 2500 cycles of the steepest descent method followed by 2500 cycles of the conjugate gradient method. Production runs were performed by the GPU-accelerated pmemd implementation of the sander protocol with a 1 fs time-step, using the ff14SB force field [42], and periodic boundary conditions. The temperature was controlled by a Langevin thermostat with a collision frequency of $3 \mathrm{ps}^{-1}$. Covalent bonds to hydrogen were constrained by SHAKE. The structures were minimized before being subjected to a $200 \mathrm{~ns}$ simulation run at $298 \mathrm{~K}$. PDB files were generated from the $200 \mathrm{~ns}$ simulated structures and were visualized to identify the first and last $\alpha$-helix-forming residues for Tpm fragments, Tpm1.164-154 and Tpm3.12 $65-155$. For each Tpm fragment (wild-type and mutated), the distance (end-to-end distance) between these residues was determined over the course of $200 \mathrm{~ns}$ for both chains in the coiled coils using the corresponding trajectory and topology files. The same files were used to create movies for the $200 \mathrm{~ns}$ simulation run using UCSF Chimera.

\subsection{Statistical Analysis}

Statistical analysis for CD data was performed using paired (to compare wild-type with the mutants) and unpaired t-test (to compare results with and without DTT). Obtained $p$-values were less than 0.0003 (most being even less than 0.00001 ) demonstrating the difference is extremely statistically significant.

\section{Conclusions}

We can conclude that specific conformations adopted by period 3 of Tpm isoforms 3.12 and 1.1 define many Tpm interactions and, consequently, the performance of the thin filament. Disease-causing mutations disrupt the structure of the Tpm coiled coil; however, local destabilization caused by some mutations may not be detected in full-length Tpms. Using designed Tpm fragments allowed us to determine even slight changes in the stability of the actin-binding domain 3 caused by the mutations.

Supplementary Materials: The following are available online, Figure S1: Effects of mutations on Tpm1.1 $1_{64-154}$ and Tpm3.12 $255-155$ structures. Figure S2: Effects of mutations on the flexibility of Tpm1.1 $164-154$ and Tpm3.1265-155. Distance between C $\alpha$ atoms of the first and last $\alpha$ helical residues for chains A and B of Tpm fragments, measured over the course of a 200 ns MDS run. Figure S3: End-to-end distance between $\mathrm{C} \alpha$ atoms of the first and last $\alpha$-helical residues for Chains $\mathrm{A}$ and $\mathrm{B}$ of Tpm fragments Tpm1.164-154 (A) and Tpm1.1 $1_{64-154}$ I92T (B) with changed side chain positions, measured over the course of a 200 ns MDS run. Table S1: The average end-to-end distances of Tpm fragments.

Author Contributions: Conceptualization, J.M. and A.S.K.; methodology, M.K.-B., J.M., B.K. and M.Ś.; investigation, B.K., M.K., M.F. and M.Ś.; resources, J.M. and A.S.K.; writing一original draft 
preparation, B.K.; writing-review and editing, J.M. and A.S.K.; supervision, J.M. and A.S.K.; project administration, J.M. and A.S.K.; funding acquisition, J.M. and A.S.K. All authors have read and agreed to the published version of the manuscript.

Funding: This work was funded by the RO1 grant from the National Institutes of Health GM120137 to A.S.K., National Science Center, grant number 2014/15/B/NZ1/01017 and by the Polish Minister of Science and Higher Education, under the program "Regional Initiative of Excellence" in 2019-2022 (Grant No. 008/RID/2018/19).

Institutional Review Board Statement: Not applicable.

Informed Consent Statement: Not applicable.

Data Availability Statement: The data presented in this study are available from the authors.

Conflicts of Interest: The authors declare no conflict of interest.

Sample Availability: Samples of the cDNA of tropomyosin peptides are available from the authors.

\section{References}

1. Hitchcock-DeGregori, S.E.; Barua, B. Tropomyosin Structure, Function, and Interactions: A Dynamic Regulator. Fibrous Proteins Struct. Mech. 2017, 82, 253-284. [CrossRef]

2. Gordon, A.M.; Homsher, E.; Regnier, M. Regulation of contraction in striated muscle. Physiol. Rev. 2000, 80, 853-924. [CrossRef]

3. Geeves, M.A.; Hitchcock-DeGregori, S.E.; Gunning, P.W. A systematic nomenclature for mammalian tropomyosin isoforms. J. Muscle Res. Cell Motil. 2014, 36, 147-153. [CrossRef]

4. Moraczewska, J. Thin filament dysfunctions caused by mutations in tropomyosin Tpm3.12 and Tpm1.1. J. Muscle Res. Cell. Motil. 2020, 41, 39-53. [CrossRef] [PubMed]

5. Matyushenko, A.M.; Shchepkin, D.V.; Kopylova, G.V.; Bershitsky, S.Y.; Levitsky, D.I. Unique functional properties of slow skeletal muscle tropomyosin. Biochimie 2020, 174, 1-8. [CrossRef] [PubMed]

6. Robaszkiewicz, K.; Sliwinska, M.; Moraczewska, J. Regulation of Actin Filament Length by Muscle Isoforms of Tropomyosin and Cofilin. Int. J. Mol. Sci. 2020, 21, 4285. [CrossRef]

7. Hitchcock-DeGregori, S.E. Tropomyosin: Function follows structure. Adv. Exp. Med. Biol. 2008, 644, 60-72.

8. Barua, B. Periodicities designed in the tropomyosin sequence and structure define its functions. Bioarchitecture 2013, 3, 51-56. [CrossRef]

9. Barua, B.; Pamula, M.C.; Hitchcock-DeGregori, S.E. Evolutionarily conserved surface residues constitute actin binding sites of tropomyosin. Proc. Natl. Acad. Sci. USA 2011, 108, 10150-10155. [CrossRef]

10. Li, X.E.; Tobacman, L.S.; Mun, J.Y.; Craig, R.; Fischer, S.; Lehman, W. Tropomyosin position on F-actin revealed by EM reconstruction and computational chemistry. Biophys. J. 2011, 100, 1005-1013. [CrossRef]

11. Hitchcock-DeGregori, S.E.; Song, Y.; Greenfield, N.J. Functions of tropomyosin's periodic repeats. Biochemistry 2002, 41, 1503615044. [CrossRef]

12. Singh, A.; Hitchcock-DeGregori, S.E. Tropomyosin's periods are quasi-equivalent for actin binding but have specific regulatory functions. Biochemistry 2007, 46, 14917-14927. [CrossRef]

13. Behrmann, E.; Muller, M.; Penczek, P.A.; Mannherz, H.G.; Manstein, D.J.; Raunser, S. Structure of the rigor actin-tropomyosinmyosin complex. Cell 2012, 150, 327-338. [CrossRef]

14. Doran, M.H.; Pavadai, E.; Rynkiewicz, M.J.; Walklate, J.; Bullitt, E.; Moore, J.R.; Regnier, M.; Geeves, M.A.; Lehman, W. Cryo-EM and Molecular Docking Shows Myosin Loop 4 Contacts Actin and Tropomyosin on Thin Filaments. Biophys. J. 2020, 119, 821-830. [CrossRef]

15. Barua, B.; Winkelmann, D.A.; White, H.D.; Hitchcock-DeGregori, S.E. Regulation of actin-myosin interaction by conserved periodic sites of tropomyosin. Proc. Natl. Acad. Sci. USA 2012, 109, 18425-18430. [CrossRef]

16. Landis, C.; Back, N.; Homsher, E.; Tobacman, L.S. Effects of tropomyosin internal deletions on thin filament function. J. Biol. Chem. 1999, 274, 31279-31285. [CrossRef]

17. Landis, C.A.; Bobkova, A.; Homsher, E.; Tobacman, L.S. The active state of the thin filament is destabilized by an internal deletion in tropomyosin. J. Biol. Chem. 1997, 272, 14051-14056. [CrossRef]

18. Singh, A.; Hitchcock-DeGregori, S.E. Dual requirement for flexibility and specificity for binding of the coiled-coil tropomyosin to its target, actin. Structure 2006, 14, 43-50. [CrossRef]

19. Singh, A.; Hitchcock-Degregori, S.E. A peek into tropomyosin binding and unfolding on the actin filament. PLoS ONE 2009, 4, e6336. [CrossRef]

20. Hitchcock-DeGregori, S.E.; An, Y. Integral repeats and a continuous coiled coil are required for binding of striated muscle tropomyosin to the regulated actin filament. J. Biol. Chem. 1996, 271, 3600-3603. [CrossRef]

21. Kawai, M.; Lu, X.; Hitchcock-Degregori, S.E.; Stanton, K.J.; Wandling, M.W. Tropomyosin period 3 is essential for enhancement of isometric tension in thin filament-reconstituted bovine myocardium. J. Biophys. 2009, 2009, 380967. [CrossRef] 
22. Oguchi, Y.; Ishizuka, J.; Hitchcock-DeGregori, S.E.; Ishiwata, S.; Kawai, M. The role of tropomyosin domains in cooperative activation of the actin-myosin interaction. J. Mol. Biol. 2011, 414, 667-680. [CrossRef]

23. Marttila, M.; Lehtokari, V.L.; Marston, S.; Nyman, T.A.; Barnerias, C.; Beggs, A.H.; Bertini, E.; Ceyhan-Birsoy, O.; Cintas, P.; Gerard, M.; et al. Mutation update and genotype-phenotype correlations of novel and previously described mutations in TPM2 and TPM3 causing congenital myopathies. Hum. Mutat. 2014, 35, 779-790. [CrossRef]

24. Redwood, C.; Robinson, P. Alpha-tropomyosin mutations in inherited cardiomyopathies. J. Muscle Res. Cell. Motil. 2013, 34, 285-294. [CrossRef]

25. Karibe, A.; Tobacman, L.S.; Strand, J.; Butters, C.; Back, N.; Bachinski, L.L.; Arai, A.E.; Ortiz, A.; Roberts, R.; Homsher, E.; et al. Hypertrophic cardiomyopathy caused by a novel alpha-tropomyosin mutation (V95A) is associated with mild cardiac phenotype, abnormal calcium binding to troponin, abnormal myosin cycling, and poor prognosis. Circulation 2001, 103, 65-71. [CrossRef]

26. Hershberger, R.E.; Norton, N.; Morales, A.; Li, D.; Siegfried, J.D.; Gonzalez-Quintana, J. Coding sequence rare variants identified in MYBPC3, MYH6, TPM1, TNNC1, and TNNI3 from 312 patients with familial or idiopathic dilated cardiomyopathy. Circ. Cardiovasc. Genet. 2010, 3, 155-161. [CrossRef]

27. Lawlor, M.W.; Dechene, E.T.; Roumm, E.; Geggel, A.S.; Moghadaszadeh, B.; Beggs, A.H. Mutations of tropomyosin 3 (TPM3) are common and associated with type 1 myofiber hypotrophy in congenital fiber type disproportion. Hum. Mutat. 2010, 31, 176-183. [CrossRef]

28. Shapovalov, M.V.; Dunbrack, R.L., Jr. A smoothed backbone-dependent rotamer library for proteins derived from adaptive kernel density estimates and regressions. Structure 2011, 19, 844-858. [CrossRef]

29. Matyushenko, A.M.; Kleymenov, S.Y.; Susorov, D.S.; Levitsky, D.I. Thermal unfolding of homodimers and heterodimers of different skeletal-muscle isoforms of tropomyosin. Biophys. Chem. 2018, 243, 1-7. [CrossRef]

30. Gonchar, A.D.; Kopylova, G.V.; Kochurova, A.M.; Berg, V.Y.; Shchepkin, D.V.; Koubasova, N.A.; Tsaturyan, A.K.; Kleymenov, S.Y.; Matyushenko, A.M.; Levitsky, D.I. Effects of myopathy-causing mutations R91P and R245G in the TPM3 gene on structural and functional properties of slow skeletal muscle tropomyosin. Biochem. Biophys. Res. Commun. 2021, 534, 8-13. [CrossRef]

31. Matyushenko, A.M.; Artemova, N.V.; Sluchanko, N.N.; Levitsky, D.I. Effects of two stabilizing substitutions, D137L and G126R, in the middle part of alpha-tropomyosin on the domain structure of its molecule. Biophys. Chem. 2015, 196, 77-85. [CrossRef]

32. Moraczewska, J.; Robaszkiewicz, K.; Śliwinska, M.; Czajkowska, M.; Ly, T.; Kostyukova, A.; Wen, H.; Zheng, W. Congenital myopathy-related mutations in tropomyosin disrupt regulatory function through altered actin affinity and tropomodulin binding. FEBS J. 2019, 286, 1877-1893. [CrossRef]

33. Letai, A.; Coulombe, P.A.; Fuchs, E. Do the ends justify the mean? Proline mutations at the ends of the keratin coiled-coil rod segment are more disruptive than internal mutations. J. Cell Biol. 1992, 116, 1181-1195. [CrossRef]

34. Ly, T.; Krieger, I.; Tolkatchev, D.; Krone, C.; Moural, T.; Samatey, F.A.; Kang, C.; Kostyukova, A.S. Structural destabilization of tropomyosin induced by the cardiomyopathy-linked mutation R21H. Protein Sci. 2018, 27, 498-508. [CrossRef]

35. Demers, J.P.; Mittermaier, A. Binding mechanism of an SH3 domain studied by NMR and ITC. J. Am. Chem. Soc. 2009, 131, 4355-4367. [CrossRef]

36. Xue, Y.; Yuwen, T.; Zhu, F.; Skrynnikov, N.R. Role of electrostatic interactions in binding of peptides and intrinsically disordered proteins to their folded targets. 1. NMR and MD characterization of the complex between the c-Crk N-SH3 domain and the peptide Sos. Biochemistry 2014, 53, 6473-6495. [CrossRef]

37. Potekhin, S.A.; Privalov, P.L. Co-operative blocks in tropomyosin. J. Mol. Biol. 1982, 159, 519-535. [CrossRef]

38. Itzhaki, R.F.; Gill, D.M. A Micro-Biuret Method for Estimating Proteins. Anal. Biochem. 1964, 9, 401-410. [CrossRef]

39. Pettersen, E.F.; Goddard, T.D.; Huang, C.C.; Couch, G.S.; Greenblatt, D.M.; Meng, E.C.; Ferrin, T.E. UCSF Chimera-A visualization system for exploratory research and analysis. J. Comput. Chem. 2004, 25, 1605-1612. [CrossRef]

40. Jorgensen, W.L.; Chandrasekhar, J.; Madura, J.D.; Impey, R.W.; Klein, M.L. Comparison of simple potential functions for simulating liquid water. J. Chem. Phys. 1983, 79, 926-935. [CrossRef]

41. Lee, T.S.; Cerutti, D.S.; Mermelstein, D.; Lin, C.; LeGrand, S.; Giese, T.J.; Roitberg, A.; Case, D.A.; Walker, R.C.; York, D.M. GPU-Accelerated Molecular Dynamics and Free Energy Methods in Amber18: Performance Enhancements and New Features. J. Chem. Inf. Model. 2018, 58, 2043-2050. [CrossRef]

42. Maier, J.A.; Martinez, C.; Kasavajhala, K.; Wickstrom, L.; Hauser, K.E.; Simmerling, C. ff14SB: Improving the Accuracy of Protein Side Chain and Backbone Parameters from ff99SB. J. Chem. Theory Comput. 2015, 11, 3696-3713. [CrossRef] [PubMed] 